\title{
Single Molecular Anatomy of Solvophobic Effects in Host-Guest Interactions Based on Surface Tension Using Atomic Force Microscopy
}

\author{
Satoshi Yasuda, ${ }^{1}$ Iwao Suzuki, ${ }^{2}$ Ken-ichi Shinohara, ${ }^{3}$ and Hidemi Shigekawa ${ }^{1, *}$ \\ ${ }^{1}$ Institute of Applied Physics, 21st Century COE, CREST (JST), University of Tsukuba, Tsukuba, Ibaraki 305-8573, Japan \\ ${ }^{2}$ Graduate School of Pharmaceutical Sciences, Tohoku University, Sendai 980-8578, Japan \\ ${ }^{3}$ School of Materials Science, Japan Advanced Institute of Science and Technology, Nomi, Ishikawa 923-1292, Japan
}

(Received 20 September 2005; published 8 June 2006)

\begin{abstract}
The effectiveness of the mechanism of surface tension to analyze the host-guest interaction was demonstrated by measuring the unbinding force of the $\beta$-Cyclodextrin $(\mathrm{CyD})$ - adamantane molecules in a liquid environment at the single-molecule level. The contribution of the solvophobic effect was quantitatively distinguished from the intrinsic van der Waals force, and the effective length that produces the detected force through employing the notion of surface tension was determined to be $1.07 \pm 0.13 \mathrm{~nm}$, which is consistent with the perimeter of the adamantane molecule, $\sim 1.3 \mathrm{~nm}$. Specific strong interaction of water molecules was also confirmed on the nanoscale.
\end{abstract}

PACS numbers: $82.65 .+\mathrm{r}, 82.33 .-\mathrm{z}$

A barberpole or a sailboat floating on water, capillary action, formation of droplets, and soap bubbles: these are familiar sights in our daily life. Behind these immediate surroundings is surface tension. Surface tension has a 200year-old scientific history since its basis was first presented by T. Young in 1805 [1]. The concept of "surface tension" was introduced and defined in the paper as the force acting on a hypothetical line of unit length arbitrarily drawn on the surface of a liquid. With the development of analytical techniques in quantum optics and surface science, this concept has been applied to the understanding of physical and chemical properties at even down to the nanoscale. For example, formation of gas phase clusters and the dynamics of thin film growth have been extensively studied and understood, generating surface tension arguments. Fine control of nanometer sized structures known as quantum dots based on such results, for instance, is one of the keys to current science and technology [2].

In this Letter, we demonstrate the effectiveness of employing the notion of surface tension for the analysis of a host-guest interaction system in a liquid. Host-guest chemistry is another key to the future of nanotechnology and biotechnology applications, and its recent progress is remarkable $[3,4]$. However, the detailed design of host-guest systems, which exhibits desired functions in predetermined structures, has not yet been successful. The most critical point is that the processes include complicated many-body effects arising from solvent-related factors. Since most important systems are effective in a liquid, interactions arising from solvent-related factors, such as the solvophobic effect, play important roles in intermolecular dynamics in addition to intrinsic forces such as van der Waals (vdW) interaction and hydrogen bonding [5-7]. Therefore, a greater understanding of the influence of the solvent effect upon the stability or association of a complex system is very important to realize the desired host-guest systems.

With the development of atomic force microscopy (AFM) [8], it is possible to directly measure the interac- tions between individual molecules at the pico-Newton level [9-20]. Using this technique, we show the first direct evidence that the mechanism of the surface tension works in the host-guest system at the single-molecule level. We have succeeded in distinguishing the solvophobic effect in the host-guest interaction from the intrinsic vdW force at the single-molecule level. The specific force of water due to hydrogen bonding is also shown to be effective on the nanoscale.

Placed on the water surface in a glass, aluminum coins sink when their weight exceeds the force produced by the surface tension of the water. The maximum weight that breaks the force balance can be related to surface tension. For the analysis of a similar experiment on the nanoscale, we adopted a host-guest molecular system, a combination of $\beta$-cyclodextrin $(\beta$-CyD) and adamantane molecules. $\beta$-CyD is a doughnut-shaped oligosaccharide consisting of seven glucose units linked in a cyclic form [Fig. 1(a)] and forms an inclusion complex with an adamantane molecule. The inside and outside diameters of $\beta$-CyD molecule are $0.7 \mathrm{~nm}$ and $1.5 \mathrm{~nm}$, respectively [3,4]. Therefore, the measurement of the unbinding force of the complex in a liquid environment enables us to analyze the surface tension of a solvent on the nanoscale. Namely, if the included admantane molecule is pulled out of the CyD cavity in a liquid environment, the detected unbinding force can be related to the surface tension of the liquid similarly seen in the case of the aluminum coins mentioned above.

Since the binding force of this system $(\beta-\mathrm{CyD}$ and adamantane molecules) arises from the vdW and hydrophobic interactions and is free from hydrogen bonding [21] and charge transfer, the analysis of force-measurement results is simple and reliable. This host-guest system is also an excellent model for the understanding of the solvophobic effect in the protein-ligand enzyme interactions because its association constant is similar to those of many protein-ligand systems [22]. 
(a)

(b)
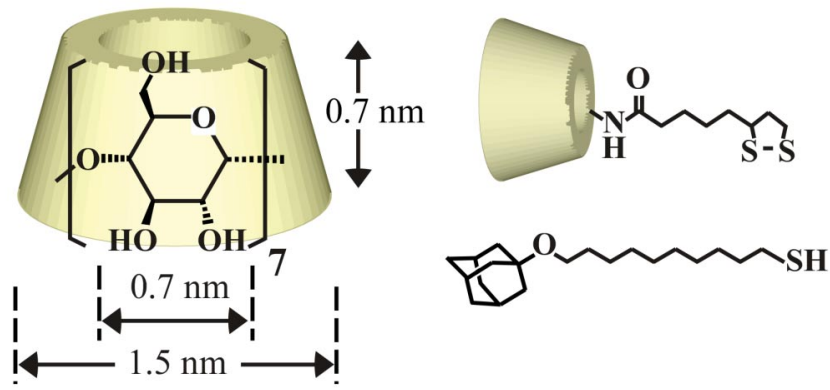

(c)

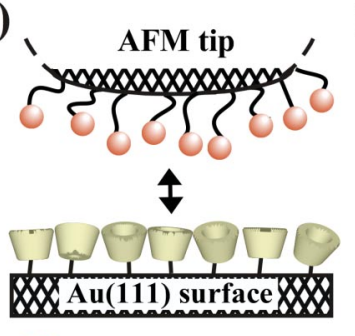

: Adamantane

: $\beta-C y D$ (d)

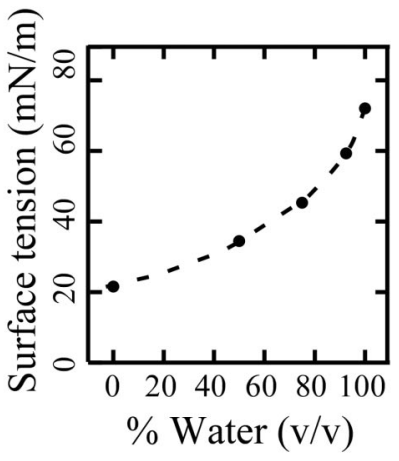

FIG. 1 (color). Structures of (a) $\beta$-cyclodextrin (CyD), (b) LP- $\beta$-CyD and 10-adamantanyloxydecane-1-thiol molecules, and (c) schematic illustration of experimental conformation. (d) Relationship between surface tension and volume ratio of methanol-water solutions.

To determine the binding force between individual $\beta$-CyD and adamantane molecules, the adhesion force between an AFM cantilever functionalized using adamantane and self-assembled monolayers (SAMs) of $\beta$-CyD was measured and analyzed. To form the SAMs of $\beta$-CyD molecules on a $\mathrm{Au}(111)$ surface, we used LP- $\beta$-CyD molecules, which are $\beta$-CyD molecules modified with a lipoamide residue chemically bound to the primary hydroxyl side. The quality of the structure of the densely packed homogeneous LP- $\beta$-CyD monolayer on the $\mathrm{Au}(111)$ surface was examined and confirmed using scanning tunneling microscopy and cyclic voltammogram measurements $[23,24]$. The adamantane molecules were chemically bonded (tethered) on a gold-coated $\mathrm{Si}_{3} \mathrm{~N}_{4} \mathrm{AFM}$ cantilever using 10-adamantanyloxydecane-1-thiol molecules, which are adamantane molecules modified with an octanethiol chemically added to one adamantyl group. The schematics of the modified molecules and the experimental conformations of the molecules are shown in Figs. 1(b) and $1(\mathrm{c})$, respectively.

For the analysis of surface tension on the nanoscale, binding-force measurement was performed in methanolwater solutions of various volume ratios. The solutions with different volume ratios provide different surface tensions. The relationship between the surface tension and the volume ratio of the methanol-water solutions in Fig. 1(d) is determined by macroscopic measurement [25].

A noteworthy point is that the macroscopically obtained surface tension of water is larger than that expected from the linear relationship from the mix ratio of the methanolwater solutions. This is because water molecules form a specific network structure that is considered to induce an additional effect. The network has been analyzed macroscopically and recognized as the reason for the high surface tension, high specific heat, and high evaporation heat of water. Whether this structure is on the nanoscale or not is another interesting issue that should be resolved.

Figure 2(a) shows the typical force curve obtained. Unbinding events occurred step by step, as shown in Fig. 2(a). This is because the tip-sample bonding consists of several individual host-guest bondings. For the analysis of the host-guest interaction, the pull-off force in the final step, indicated by "F" in Fig. 2(a), was recorded. More than 300 force measurements were performed for each solvent. A histogram of the pull-off forces measured for water is shown in Fig. 2(b). As indicated by arrows, there exists a certain periodic feature in the histogram, which is more clearly confirmed using the autocorrelation analysis of the histogram, as shown in Fig. 2(c) [26,27]. This result indicates that the obtained pull-off forces are composed of integer multiples of an elementary force for unbinding a single $\beta$-CyD-adamantane complex, which is determined to be $97 \pm 12 \mathrm{pN}$ in the present case. This value is close to that obtained in the previous work, $102 \pm 15 \mathrm{pN}$ [17]. Through a similar procedure, the unbinding forces in all solutions were determined. The measurement error (12\%) mainly arises from that in the calibration of the spring constant of the cantilevers [28].

\section{(a)}

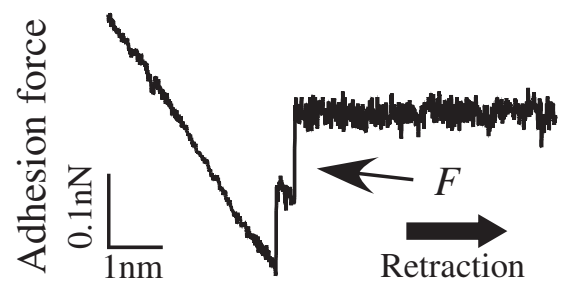

Tip displacement

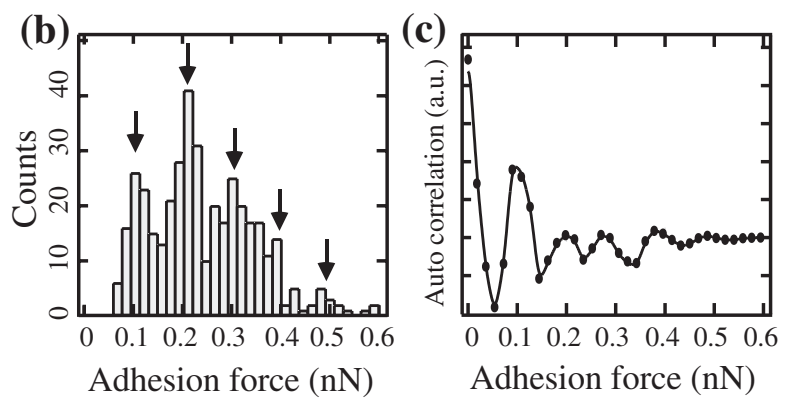

FIG. 2. (a) Typical force curve obtained for water. (b) Histogram derived from the force measurement in water, and (c) autocorrelation analysis of histogram shown in (b). 
Figure 3(a) shows the obtained unbinding force plotted as a function of the surface tension of the solvent, which was determined by a macroscopic method [Fig. 1(d)] [25]. The relationship between the unbinding force and the surface tension could be indicative of parameters such as intermolecular interaction forces. To reduce the effect of the complicated interactions, the host-guest system was chosen, as mentioned before, because the binding force arises from the vdW and hydrophobic interactions and is free from hydrogen bonding and charge transfer. Therefore, the detected unbinding force is related to the surface tension of the liquid, the solvent-solvent interaction.

As presented in Fig. 3(a), the unbinding force linearly increases with the increasing surface tension of the solvents, that is, the unbinding force is proportional to the surface tension of the solvent. Since surface tension is defined as the force acting on a hypothetical line of unit length arbitrarily drawn on the liquid surface, the obtained linear relationship indicates that there exists a specific "effective length" that produces the observed unbinding force; surface tension = [(unbinding force)-(vdW force)]/(effective length). From the gradient of the linear relationship shown in Fig. 3(a), the effective length is estimated to be $1.07 \pm 0.13 \mathrm{~nm}$, which is consistent with the perimeter of the adamantane molecule, $\sim 1.3 \mathrm{~nm}$. This is the first direct evidence showing that surface tension works in the host-guest system at the single-molecule level.

From the linear relationship shown in Fig. 3(a), the intrinsic $\mathrm{vdW}$ force can be determined by extrapolating the linear relationship to zero surface tension, and the value is $18 \pm 7 \mathrm{pN}$; this makes the comparison with the theoretical analysis possible, for example, using the structure determined by the x-ray diffraction method. By subtracting the vdW force from the measured force, the force caused by the surface tension for each liquid can be evaluated. This is also the first time that the factors contributing to the host-guest interaction in a liquid environment are discriminated at the single-molecule level. According to the macroscopic analysis, the $\mathrm{vdW}$ force component is about
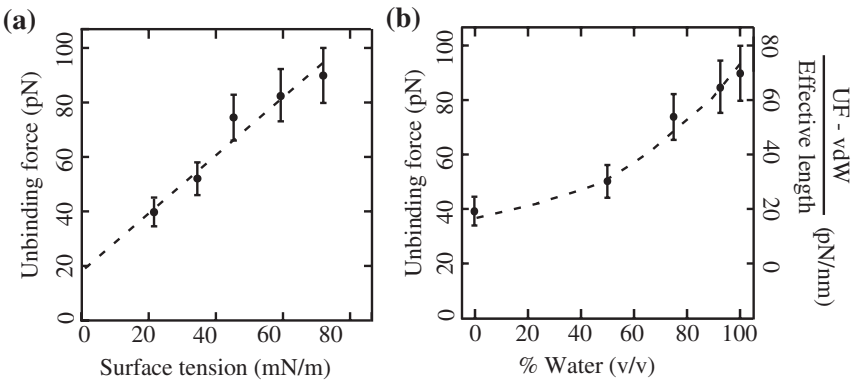

FIG. 3. Obtained unbinding forces as a function of surface tension of solvents (a) and as a function of the mix ratios of the methanol-water solutions (b). Right side axis represents the surface tensions determined using AFM measurement, i.e., unbinding force (UF) minus vdW force divided by effective length.
$70 \%$ of the total force [29], while it is $25 \%$ (100\% water) $50 \%$ (100\% methanol) in this case, as shown in Fig. 3(a). This can be attributed to the fact that the vdW force measured here is just on the unbinding process of the complex structure, where vdW force is weaker than that for the stable conformation (Fig. 4).

The linear relationship in Fig. 3(a) indicates that the surface tension determined by AFM measurement, that is, unbinding force minus vdW force divided by the effective length, has the same function of the mix ratios of the methanol-water solutions as the macroscopic relationship shown in Fig. 1(d). To further elucidate the relationship of the nanoscale mechanism with the macroscopic mechanism, the unbinding force obtained using AFM measurement is plotted as a function of the mix ratios of the methanol-water solutions in Fig. 3(b). Here, it is clearly shown that the characteristic of the solvophobic effect obtained on the nanoscale, namely, the unbinding force minus vdW $(18 \pm 7 \mathrm{pN})$ divided by the effective length $(1.07 \pm 0.13 \mathrm{~nm})$, is in good agreement with that of the macroscopic surface tension of the solution shown in Fig. 1(d).

As mentioned earlier, the surface tension of water is larger than that expected from the linear relationship from the mix ratio of the methanol-water solutions, indicating that the interaction between water molecules is stronger than those between molecules of other solvents, which is considered to be induced by the strong hydrogen bond network [30]. Our result is the first demonstration that shows the existence of a specific network of water molecules in the host-guest system, which provides us with the basis of the results of the macroscopic analysis.

In conclusion, the effectiveness of the mechanism of surface tension on the host-guest system was demonstrated at the single-molecule level by measuring the unbinding force in a liquid environment. The contribution of surface tension was quantitatively distinguished from the intrinsic $\mathrm{vdW}$. The effective length that produces the detected force through the mechanism of surface tension was determined to be $1.07 \pm 0.13 \mathrm{~nm}$, which is consistent with the perimeter of the adamantane molecule, $\sim 1.3 \mathrm{~nm}$. Specific strong

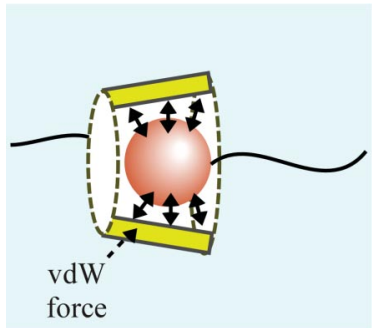

Complex

structure

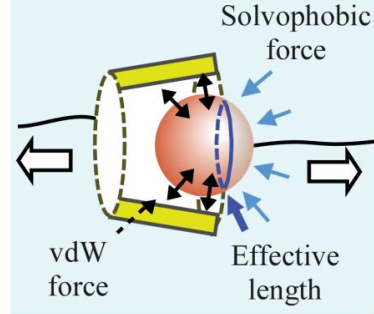

Unbinding process
FIG. 4 (color). Schematic illustrations showing the difference in $\mathrm{vdW}$ forces appearing in stable and unbinding-process conformations. 
interaction of water molecules was also confirmed on the nanoscale.

This work was supported by a Grant-in-Aid for Scientific Research from the Ministry of Education, Culture, Sports, Science, and Technology of Japan. One of the authors (S. Y.) was financially supported by the Japan Society for the Promotion of Science (JSPS). We thank Ms. Rie Yamashita for her help in preparing this Letter.

*Electronic address: http://dora.ims.tsukuba.ac.jp

[1] T. Young, Phil. Trans. Roy. Soc. London 95, 65 (1855).

[2] T. Someya, R. Wermer, A. Forchel, M. Catalano, R. Cingolani, and Y. Arakawa, Science 285, 1905 (1999).

[3] J.M. Lehn,Comprehensive Supramolecular Chemistry, Cyclodextrins (Elsevier, Oxford, 1996), Vol. 3.

[4] A. Harada, Acc. Chem. Res. 34, 456 (2001).

[5] O. Sinanoğlu, Molecular Associations in Biology, edited by B. Pullman (Academic, New York, 1968), p. 427.

[6] O. Sinanoğlu and A. Fernandez, Biophys. Chem. 21, 167 (1985).

[7] J. Harrison and M. Eftink, Biopolymers 21, 1153 (1982).

[8] G. Binning, C. F. Quate, and C. Gerber, Phys. Rev. Lett. 56, 930 (1986).

[9] G. U. Lee, L. A. Chrisey, and R. J. Colton, Science 266, 771 (1994).

[10] G. U. Lee, D. A. Kidwell, and R. J. Colton, Langmuir 10, 354 (1994).

[11] E.-L. Florin, V. T. Moy, and H. E. Gaub, Science 264, 415 (1994).

[12] V. T. Moy, E.-L. Florin, and H. E. Gaub, Science 266, 257 (1994).

[13] M. Rief, F. Oesterhelt, B. Heymann, and H.E. Gaub, Science 275, 1295 (1997).

[14] M. Rief, M. Gautol, F. Oesterhelt, J. M. Fornandez, and H. E. Gaub, Science 276, 1109 (1997).

[15] H. Schönherr, M.W. J. Beulen, J. Bugler, J. Huskens, F. C. J. M. Veggel, D. N. Reinhoudt, and G. J. Vancso, J. Am. Chem. Soc. 122, 4963 (2000).

[16] S. Zapotoczny, T. Auletta, M. R. Jong, H. Schönherr, J. Huskens, F. C. J. M. Veggel, D. N. Reinhoudt, and G. J. Vancso, Langmuir 18, 6988 (2002).

[17] T. Auletta, M. R. Jong, A. Mulder, F. C. J. M. Veggel, J. Huskens, D. N. Reinhoudt, S. Zou, S. Zapotoczny, H. Schönherr, G. J. Vancso, and L. Kuipers, J. Am. Chem. Soc. 126, 1577 (2004).

[18] S. S. Wong, E. Joselevich, A. T. Woolley, C. L. Cheung, and C. M. Lieber, Nature (London) 394, 52 (1998).
[19] H. Skulason and C. D. Frisbie, J. Am. Chem. Soc. 124, 15125 (2002).

[20] B. Xu, X. Xiao, and N. J. Tao, J. Am. Chem. Soc. 125, 16164 (2003).

[21] The oxygen atom of the ether bond between the adamantane molecule and the decyl-thiol molecule could form a hydrogen bond with the 14 hydoxyl groups present at the periphery of the $\mathrm{CyD}$ cage. However, since adamantane has a bulky structure and the free space around the oxygen atom of the ether is small, it is not easy to form its hydrogen bond with the bulky hydoxyl groups on the CyD. Water molecules are small and can come close to the site, but the strength of the hydrogen bonding between the oxygen and the hydroxyl group of water is weak because of the low polarity of the ether bond. In fact, the linear relationship shown in Fig. 3(a) over all volume ratios of the methanol-water solutions indicates that there exists little influence of the water molecules on the interaction.

[22] W. C. Cromwell, K. Bystrom, and M. R. Eftink, J. Phys. Chem. 89, 326 (1985)

[23] S. Yasuda, I. Suzuki, T. Nakamura, M. Matsumoto, M. Komiyama, and H. Shigekawa, Appl. Phys. Lett. 76, 643 (2000).

[24] S. Yasuda, I. Suzuki, J. Sumaoka, M. Komiyama, and H. Shigekawa, Trans. Mat. Res. Soc. Jpn. 31, 853 (2000).

[25] CRC Handbook of Chemistry and Physics (CRC Press, Boca Raton, FL, 1991-1992), 72nd ed., Vol. 6, p. 117.

[26] Binding-force measurements in each mixed solution were performed several times with different tips to confirm the reproducibility. Different tips were used for different mixed solutions. Despite these procedures, a clear linearity in Fig. 3 was obtained, supporting the validity of the analysis.

[27] Although multiple pull-off measurements include complicated processes, the histogram analysis adopted in this Letter has been supported by many results [9-20]. Broadening of the histogram in this case is attributed to the fluctuations in the measurement conditions occurring at room temperature.

[28] The spring constant of the cantilevers coated with a $50 \mathrm{~nm}$ Au film was calibrated by the thermal resonance method [J. L. Hutter and J. Bechhoefer, Rev. Sci. Instrum. 64, 1868 (1993)]. The measured spring constant varied from 0.11 to $0.25 \mathrm{~N} / \mathrm{m}$, and the values obtained from more than 20 measurements were averaged for each cantilever.

[29] J.C. Harrison and M. Eftink, Biopolymers 21, 1153 (1982).

[30] S.P. Jarvis, T. Uchihashi, T. Ishida, H. Tokumoto, and Y. Nakayama, J. Phys. Chem. B 104, 6091 (2000). 